\title{
Prevention of Paradoxical Cerebral Embolus with Protection System during Combination Right Atrial Clot Aspiration Thrombectomy and Closure of Patent Foramen Ovale
}

\author{
Jason Chiang ${ }^{1}$ Sipan Mathevosian Jamil Aboulhosn² John M Moriarty ${ }^{1}$ \\ ${ }^{1}$ Department of Radiology, Ronald Reagan UCLA Medical Center, \\ Los Angeles, California, United States \\ ${ }^{2}$ Division of Cardiology, Ronald Reagan UCLA Medical Center, Los \\ Angeles, California, United States \\ Address for correspondence Jason Chiang, MD, PhD, Department \\ of Radiology, UCLA Ronald Reagan Medical Center, 757 Westwood \\ Plaza, Suite 1638, Los Angeles, CA 90095, United States \\ (e-mail: CJChiang@mednet.ucla.edu).
}

Arab J Intervent Radiol 2021;5:52-55.

\begin{abstract}
Keywords

- thrombectomy

- neuroprotection

- patent foramen ovale

In this technical case report, we describe a 41-year-old female with a history of breast cancer who was found to have a right atrial clot attached to the tip of her Port-A-Cath. During transthoracic echocardiography to evaluate her clot, she was also noted to also have a patent foramen ovale. The decision was made to perform a simultaneous right atrial endovascular aspiration thrombectomy and patent foramen ovale closure. To minimize the risk for paradoxical embolus during clot manipulation, an intravascular embolic neuroprotection device was deployed. After the procedure, it was noted on visual inspection that the device filter contained several embolic fragments. The presence of macroscopic embolic fragments in the filter baskets highlights the role of prophylactic embolic protection when performing cardiac interventions in the setting of a patent foramen ovale, particularly in the presence of a right atrial thrombus or mass.
\end{abstract}

\section{Introduction}

Cerebral embolic stroke is a potentially devastating complication in patients with patent foramen ovale (PFO). The risk of stroke is amplified in the setting of venous thromboembolism (VTE), particularly clots in transit or presence of right atrial thrombus that requires intervention to curtail potential paradoxical embolism. Cerebral embolic stroke is also a well-known potential complication of transcatheter aortic valve repair (TAVR). ${ }^{1}$ During TAVR, atherosclerotic plaque, debris, or calcified valve remnants may fragment and embolize into the cerebral vasculature causing stroke. To mitigate the risk of stroke during TAVR, cerebral neuroprotection devices for debris capture may be used. In this case report, we describe successful implementation of the Sentinel cerebral protection system (Boston Scientific, Marlborough, Massachusetts, United States) for neuroprotection from paradoxical embolus during right atrial endovascular aspiration thrombectomy and PFO closure.

\section{Case Report}

A 41-year-old female with a history of breast cancer status post bilateral mastectomy and radiation therapy with a recently placed right indwelling Port-A-Cath presented with sudden onset arm swelling. A computed tomography angiogram was performed on her right arm and chest, showing no evidence of vascular stenosis but revealing a $3.6 \mathrm{~cm}$ right atrial clot at the catheter tip. After 3 months of therapeutic anticoagulation, it was noted on transesophageal published online June 4, 2021
DOI https://doi.org/

$10.1055 / \mathrm{s}-0041-1730117$ ISSN 2542-7075

\section{(c) 2021. The Pan Arab Interventional Radiology Society}

This is an open access article published by Thieme under the terms of the Creative Commons Attribution-NonDerivative-NonCommercial-License, permitting copying and reproduction so long as the original work is given appropriate credit. Contents may not be used for commercial purposes, or adapted, remixed, transformed or built upon. (https://creativecommons.org/licenses/by-nc-nd/4.0/).

Thieme Medical and Scientific Publishers Private Ltd. A-12, Second Floor, Sector -2, NOIDA -201301, India 
echocardiography (TEE) that her right atrial thrombus was still present and that there was also a previously unidentified PFO, with a bidirectional shunt across the atrial septum seen with contrast ultrasound. Given her extensive surgical history and comorbidities relating to her breast cancer treatment regimen, a minimally invasive strategy was pursued. A multidisciplinary discussion between interventional radiologists, cardiothoracic surgeons, and cardiologists was made to proceed with minimally invasive clot retrieval following PFO closure to minimize the risk for embolization event and allow Port-A-Cath removal.

The patient was brought to the angiography suite under general anesthesia where the intraoperative TEE demonstrated a mobile thrombus extending from the Port-A-Cath tip to the free wall of the right atrium as well as the PFO ( $\mathbf{- F i g . 1 A}$ and $\mathbf{- 1 B}$ ). Traditionally, the PFO would be closed prior to clot retrieval given concern for paradoxical embolus and distal thrombus migration into the pulmonary circulation. However, due to the size of the right atrial thrombus and the catheter manipulation required to place the closure device, the Sentinel percutaneous cerebral protection device (Boston Scientific, Marlborough, Massachusetts, United States) was utilized.

The right radial artery was accessed under ultrasound guidance and a slim $5 \mathrm{~F}$ sheath was placed followed by intraarterial infusion of heparin and nitroglycerin. The articulating sheath located in the distal end of the cerebral protection device was then rotated until the tip entered the ostium of the left common carotid artery. Tension was provided to the articulating sheath such that the curvature conformed to the brachiocephalic artery-aorta-left common carotid artery junction and pulled toward the carina between the two vessels. The proximal filter was then deployed in the brachiocephalic artery under fluoroscopic guidance to confirm filter to vessel-wall apposition ( - Fig. 2A). In this configuration, the carotid basket was subsequently deployed without complication (-Fig. 2B).

From the right common femoral vein, a multipurpose catheter and 035 Glidewire were then used to cross the PFO into the left atrium, followed by exchange for an Amplatz extra-stiff wire that was placed into the left upper lobe pulmonary vein. A 9 Fr Torque view sheath was advanced across the PFO under TEE visualization. Under fluoroscopic and echocardiographic guidance, a $35 \mathrm{~mm}$ Amplatzer PFO occluder (Abbot Inc, Minneapolis, Minnesota, United States) was deployed, with particular attention to avoid the existing large right atrial thrombus. Agitated saline injection demonstrated shunting
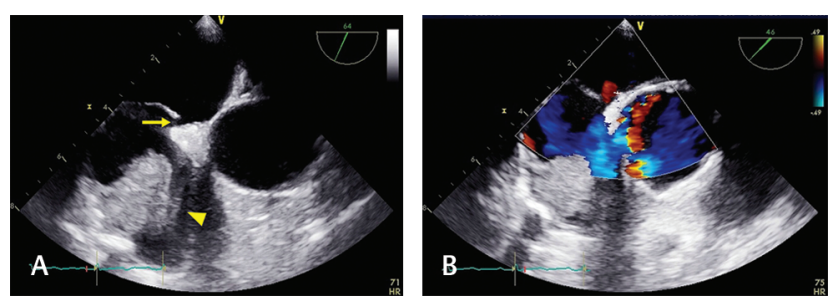

Fig. 1 Transesophageal echocardiography demonstrating (A) cross-section of a hyperechoic mobile thrombus (yellow arrowhead) abutting the free wall of the right atrium and patent foramen ovale (yellow arrow) with (B) corresponding Doppler flow present. of bubbles across the PFO at baseline was resolved following occlusion device placement and release. The Sentinel device was then withdrawn. Upon visual inspection, the Sentinel device filters contained several embolic fragments in both innominate and left common carotid artery baskets (-Fig. $\mathbf{3}$ ).

With the PFO occlusion device in place, attention was turned to the right atrium to guide removal of the right atrial thrombus under fluoroscopic and TEE guidance. Left common femoral venous access was obtained under ultrasound guidance
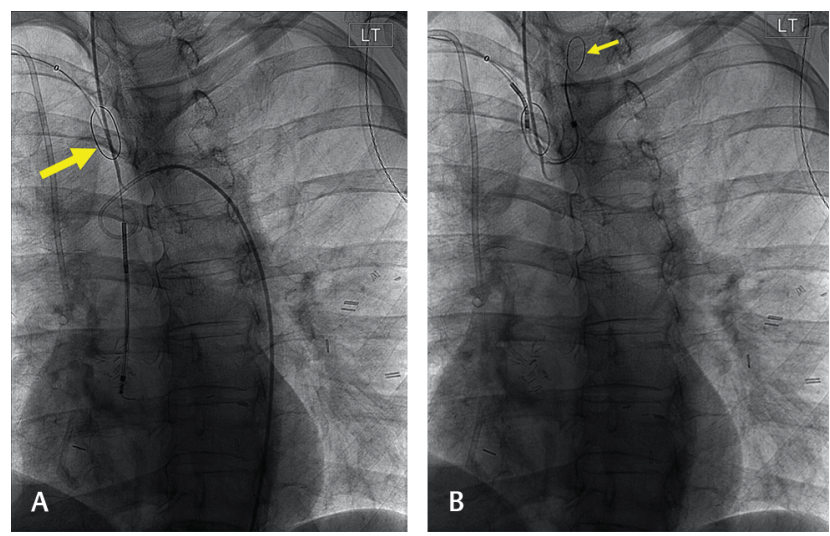

Fig. 2 (A) Proximal filter (yellow arrow) of the Sentinel system being deployed within the brachiocephalic artery over a Balance Middle Weight (BMW) wire. (B) An articulating sheath located in the distal end of the Sentinel system was advanced and rotated such that the tip could insert into the origin of the left common carotid artery. Tension was provided so that the curvature of the system was aligned with the brachiocephalic artery-aorta-left common carotid artery junction and pulled toward the carina between the two vessels. The left common carotid artery was then deployed (yellow arrow).

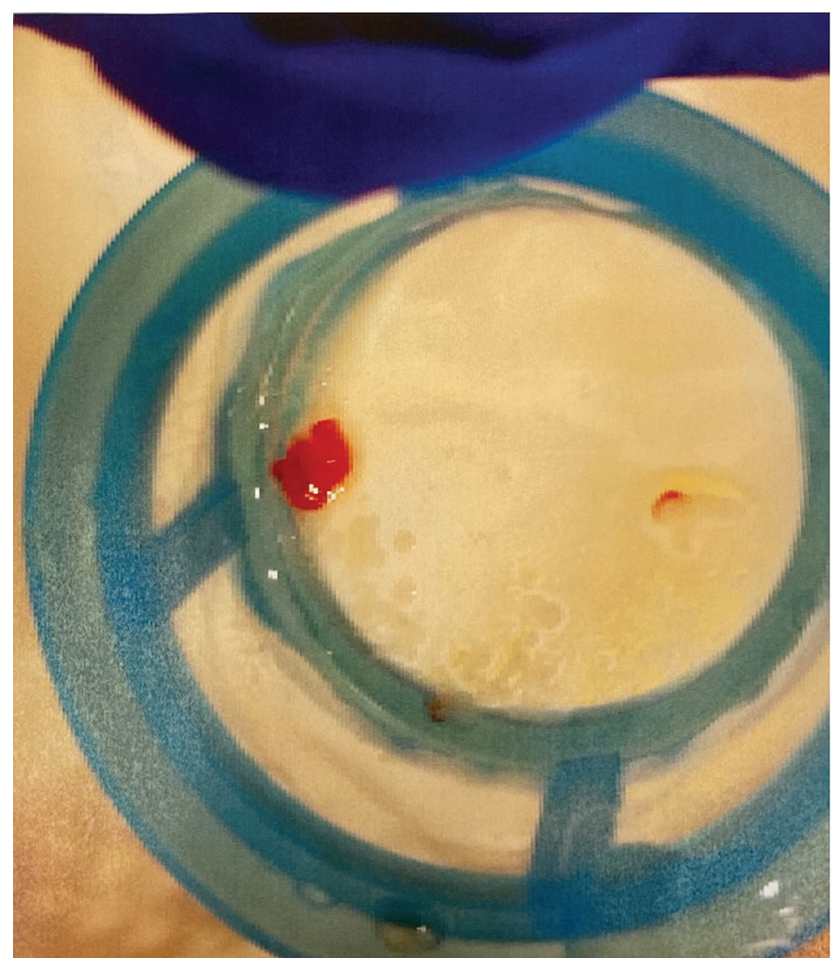

Fig. 3 Fibrous appearing chronic embolic fragments were noted in the Sentinel system filters, with a larger amount in the left common carotid basket. 
with subsequent insertion of an 18 Fr Fem-Flex reperfusion catheter advanced into the left common iliac vein. The patient was then systemically heparinized to a target activated clotting time of 300 seconds. The venovenous bypass circuit was de-aired and brought sterile to the field. Under combination of fluoroscopic and TEE guidance, the AngioVac aspiration cannula (AngioDynamics, Latham, New York, United States) was advanced into the right atrium directly against the mass, where moderate volumes of thrombus were aspirated ( - Fig. 4). On TEE, it was noted that there was residual thrombus noted abutting the free wall of the right atrium. However, no further intervention was pursued at this point due to the residual thrombus being located in proximity to the recently placed PFO closure device. After the AngioVac cannulas were removed, the port was able to be removed without complication. The postprocedure course was uneventful without evidence of neurological symptoms. While there is also a possibility of embolic material traveling distally to abdominal organs or extremities, there was no indication of critical limb ischemia or visceral organ infarct noted in this case. The patient subsequently discharged home after the procedure on apixaban $5 \mathrm{mg}$ twice daily. Follow-up transthoracic echo after 6 weeks failed to identify any significant residual right atrial thrombus.

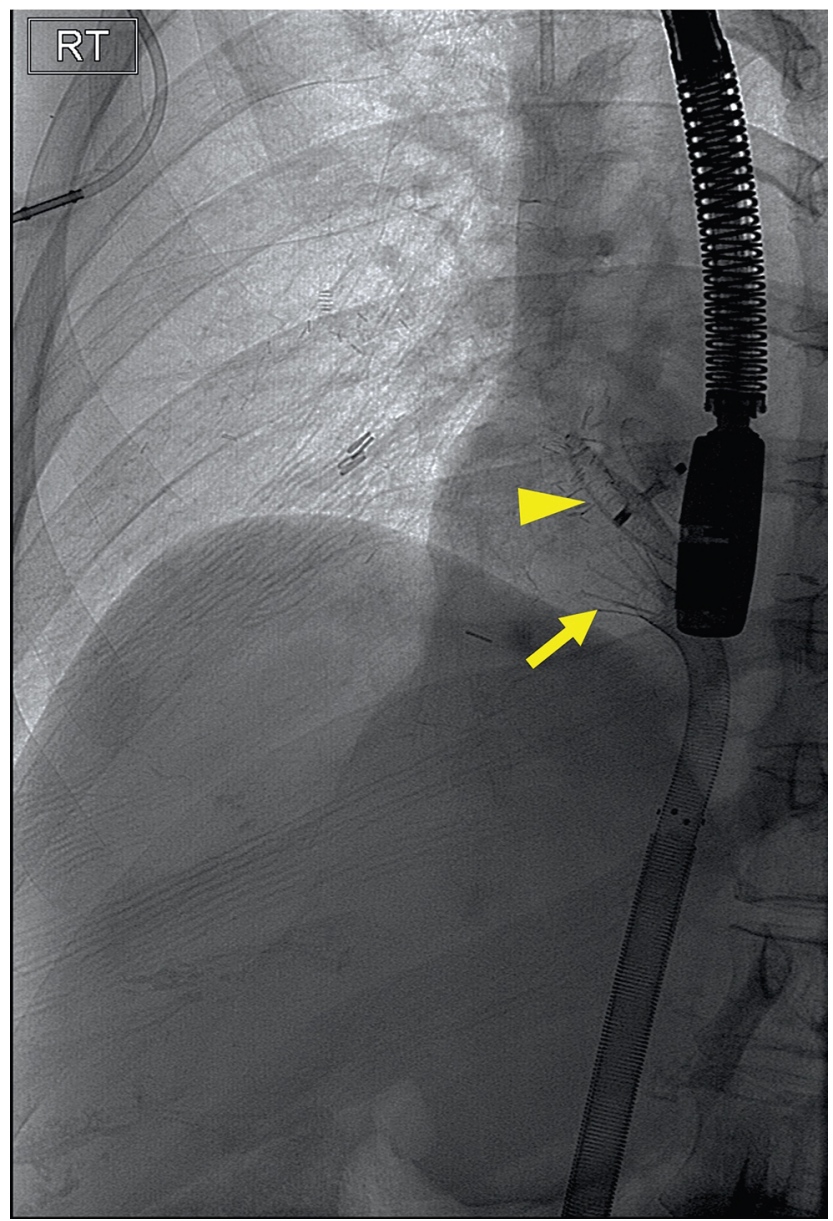

Fig. 4 Transesophageal echocardiography transducer guiding AngioVac aspiration device (yellow arrow) around the right atrial thrombus from femoral access after neuroprotection device had been deployed. The $35 \mathrm{~mm}$ closure device (yellow arrowhead) was placed prior to manipulation of the right atrial thrombus.

\section{Discussion}

Cerebral embolic stroke is a feared complication in patients undergoing aortic arch endovascular interventions. ${ }^{1}$ Apart from major stroke, microvascular "silent" infarcts are seen in approximately $70 \%$ or more of patients on postprocedure diffusion-weighted magnetic resonance imaging. ${ }^{2}$ To mitigate the risk of cerebrovascular embolization of plaque or calcified valve materials during TAVR, cerebral embolic protection devices are employed as an intraprocedural neuroprotective strategy. Our patient had a large thrombus in the right atrium with a theoretical risk of dislodgement through the PFO of similar size and into the cerebrovascular circulation. PFO closure alone without neuroprotection was thought to be of higher risk due the catheter manipulation required around a significant thrombus burden. The Sentinel neuroprotection device was therefore deployed prior to PFO closure and aspiration thrombectomy. After the procedure, the neuroprotection device was revealed to have caught multiple embolic fragments, validating the decision for stroke prevention.

Central venous catheter tip-associated thrombus is a commonly encountered scenario without clear evidence-based guidelines. The etiology of catheter-related thrombus is unknown but hypothesized to be related to mechanical irritation of the right atrial free wall or flow disruption leading to activation of coagulation pathway. ${ }^{3}$ A recent study demonstrated that out of 49 patients with echocardiogram-detected catheter-tip associated thrombus, no embolic or other complications were seen, suggesting that aggressive interventions may be unnecessary. ${ }^{4}$ In contradistinction, other groups have tried to mitigate the risk for pulmonary emboli or hemodynamic compromise by successfully reducing the clot burden with anticoagulation. ${ }^{5}$ Since our patient failed anticoagulation management, we chose to take a minimally invasive approach by removing the thrombus via a catheter-directed approach even after the PFO was closed, eliminating the risk of additional thrombus formation and potential dislodgement.

The Sentinel embolic protection device utilized in this study is a filter device designed to capture debris dislodged during TAVR, as seen in our case study. The Sentinel trial, the largest randomized clinical trial evaluating the safety and efficacy of this protection device, showed a significant reduction in median new diffusion restricting lesion volume in the protected territories up to 1 -week after TAVR. ${ }^{6}$ Furthermore, histopathological debris was found in the filters in $99 \%$ of the patients. ${ }^{6}$ A recent meta-analysis of five randomized clinical trials of 625 patients, using death or stroke as the composite endpoint, found that neuroprotective devices reduced absolute risk (ARR) of events by 3.5\% for a number-needed-to-treat (NNT) value of approximately 28. ${ }^{7}$ However, these studies largely evaluated stroke events in the short-term time frame and its use of a composite endpoint has been questioned. ${ }^{8}$ A subsequent propensity matched analysis of 560 patients utilizing dual-filter protection devices during TAVR demonstrated significant decrease in disabling and nondisabling stroke from 4.6 to $1.4 \%$ with ARR of $3.2 \%$ and NNT of 31 $(p=0.03$, odds ratio $=0.29,95 \%$ confidence interval: $0.10-0.93){ }^{9}$ 
This case demonstrates successful neuroprotection in a patient with a PFO and large right atrial thrombus prior to PFO closure and aspiration thrombectomy, with the use of the Sentinel neuroprotection device. The presence of macroscopic embolic fragments in the filter baskets highlights the role of prophylactic embolic protection when performing cardiac interventions in the setting of a PFO and elevated VTE risk, especially right atrial thrombus or mass.

\section{Disclosures}

No financial support was provided for this study.

Dr. Moriarty reports personal fees from Argon Medical, grants from AngioDynamics, personal fees from Boston Scientific, personal fees from Inari Medical, personal fees from BD Bard, personal fees from Pavmed, personal fees from Penumbra, outside the submitted work.

\section{Conflict of Interest}

None.

\section{References}

1 Smith CR, Leon MB, Mack MJ, et al; PARTNER Trial Investigators. Transcatheter versus surgical aortic-valve replacement in high-risk patients. N Engl J Med 2011;364(23):2187-2198
2 Wendt D, Kleinbongard P, Knipp S, et al. Intraaortic protection from embolization in patients undergoing transaortic transcatheter aortic valve implantation. Ann Thorac Surg 2015;100(2):686-691

3 Lok CE, Huber TS, Lee T, et al. National Kidney Foundation. KDOQI clinical practice guideline for vascular access: 2019 update. Am J Kidney Dis 2020;75(4, Suppl 2) :S1-S164

4 Chick JFB, Reddy SN, Bhatt RD, Shin BJ, Kirkpatrick JN, Trerotola SO. Significance of echocardiographically detected central venous catheter tip-associated thrombi. J Vasc Interv Radiol 2016;27(12):1872-1877

5 Yang H, Chen F, Jiao H, et al. Management of tunneled-cuffed catheter-related right atrial thrombosis in hemodialysis patients. J Vasc Surg 2018;68(5):1491-1498

6 Kapadia SR, Kodali S, Makkar R, et al. SENTINEL Trial Investigators. Protection against cerebral embolism during transcatheter aortic valve replacement. J Am Coll Cardiol 2017;69(4):367-377

7 Giustino G, Sorrentino S, Mehran R, Faggioni M, Dangas G. Cerebral embolic protection during TAVR: a clinical event meta-analysis. J Am Coll Cardiol 2017;69(4):465-466

8 Eggebrecht H, Schmermund A, Mehta RH. TAVR and stroke prevention: importance of cerebral embolic protection device data. J Am Coll Cardiol 2017;70(10):1306-1307

9 Seeger J, Gonska B, Otto M, Rottbauer W, Wöhrle J. Cerebral embolic protection during transcatheter aortic valve replacement significantly reduces death and stroke compared with unprotected procedures. JACC Cardiovasc Interv 2017;10(22):2297-2303 